\title{
Endoscopic closure of tracheoesophageal fistula with submucosal dissection and a biosynthetic mesh
}

A 67-year-old female patient was evaluated at the Hepatogastroenterology Department for the management of a chronic tracheoesophageal fistula. She had previously undergone a right lobectomy for congenital bronchiectasis, an aortic valve replacement, and a laparoscopic Heller myotomy with Toupet fundoplication for achalasia. In the past, she had also been admitted to the intensive care unit several times because of respiratory tract infections that required mechanical ventilation.

An upper endoscopy showed a 6-mm fistulous orifice in the upper third of the esophagus. Argon plasma coagulation was applied and the tract was closed using EZ clips (Olympus Medical Corp., Tokyo, Japan) and Endoloops (Olympus). Her symptoms persisted and a second treatment was attempted. A fully covered metal stent was first placed in the airway. The borders of the fistular opening were then dissected in order to create a submucosal virtual space and a mucosal flap without tension ( Video 1 ). The incision was started using a FlexKnife (Olympus), and then an IT-Knife (Olympus) was employed to continue the dissection with circular movements ( $\bullet$ Fig. 1 ) and retroflexion. A two-channel gastroscope was used

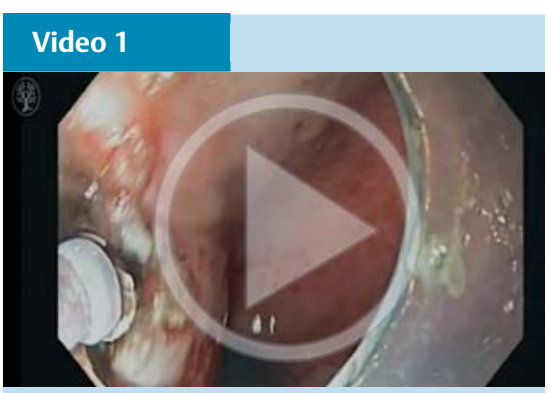

Endoscopic closure of tracheoesophageal fistula with submucosal dissection and a biosynthetic mesh. to place two round pieces of 25-mm biological mesh (Surgisis; Cook Medical, Dublin, Ireland) into the previously created space. This maneuver was performed using grasping forceps ( $\bullet$ Fig. 2). The mesh was fixed using EZ clips $90^{\circ}$ and four 20-mm Endoloops ( Fig.3), under the mucosal flap.

The patient successfully restarted oral food intake and was discharged. Upper endoscopy and bronchoscopy confirmed the closure of the orifice during the subsequent 8 months.

There are currently multiple endoscopic options available for the treatment of tracheoesophageal fistulas, with an overall success rate of $84 \%$ [1]. Only one case report has described the use of mucosal resection [2]. Biosynthetic mesh is frequently used in the surgical repair of multiple defects. However, endoscopic placement of mesh for tracheoesophageal fistula has only been described once, where it was deployed from the respiratory tract and achieved a complete closure of the orifice [3]. The case reported here describes a new endoscopic approach to tracheoesophageal fistulas that should be considered as an alternative in the management of these challenging cases.

Endoscopy_UCTN_Code_TTT_1AO_2AI

\section{Competing interests: None}

\section{lago Rodríguez-Lago ${ }^{1}$, Marc Schroeven², Daniel Rodenstein ${ }^{3}$, Pierre H. Deprez ${ }^{1}$}

${ }^{1}$ Hepatogastroenterology Department, Cliniques Universitaires St Luc, Université Catholique de Louvain, Brussels, Belgium 2 Pneumology Department, Clinique St Jean, Brussels, Belgium

${ }^{3}$ Pneumology Department, Cliniques Universitaires St-Luc, Université Catholique de Louvain, Brussels, Belgium

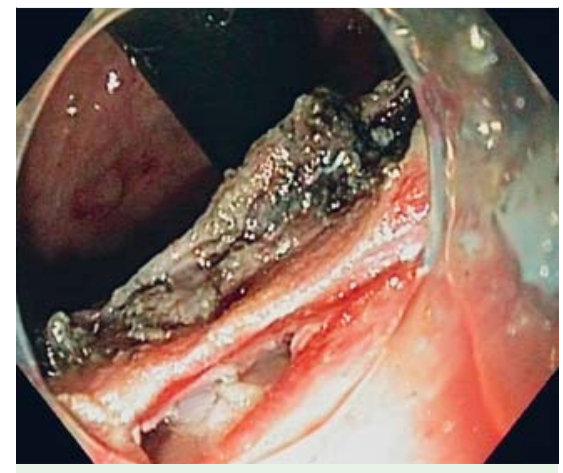

Fig. 1 Submucosal dissection of the fistulous tract in retroflexed view.

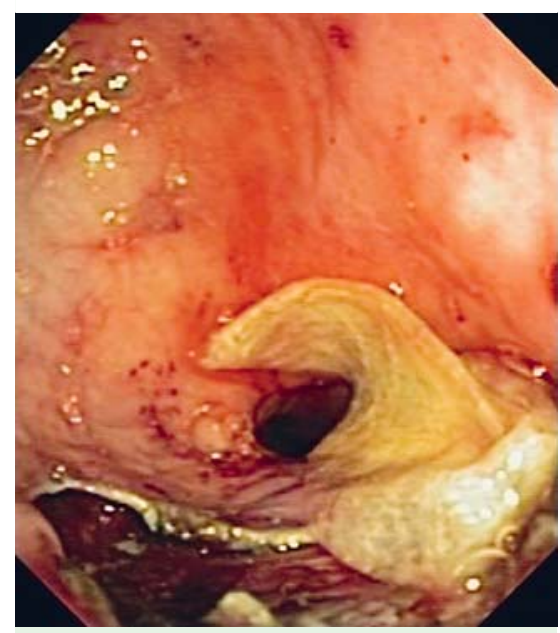

Fig. 2 Placement of a biosynthetic mesh into the virtual space created between the mucosa and submucosa.

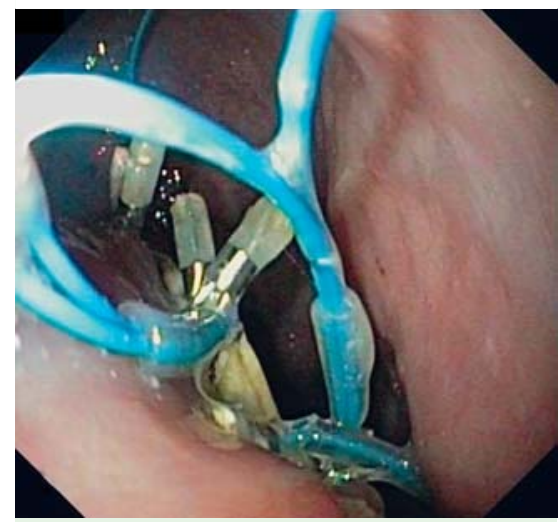

Fig. 3 Mesh fixation and closure of the orifice by placement of clips and endoloops. 


\section{References}

1 Aworanti 0 , Awadalla S. Management of recurrent tracheoesophageal fistulas: a systematic review. Eur J Pediatr Surg 2014; 24 $365-375$

$2 \mathrm{He} C \mathrm{CH}$, Zhang $\mathrm{Q}$ Liu $M$ et al. Endoscopic treatment of congenital tracheoesophageal fistula with submucosal resection and closure by clip. Can J Gastroenterol 2012; 26 : 247-248

3 Keckler SJ, St Peter SD, Calkins CM et al. Occlusion of a recurrent tracheoesophageal fistula with surgisis. J Laparoendosc Adv Surg Tech A 2008; 18: 465-468
Bibliography

DOI http://dx.doi.org/

10.1055/s-0034-1392315

Endoscopy 2015; 47: E312-E313

(c) Georg Thieme Verlag KG

Stuttgart · New York

ISSN 0013-726X
Corresponding author

Pierre H. Deprez, MD, PhD

Hepatogastroenterology Department

Cliniques Universitaires St-Luc

Université Catholique de Louvain

Avenue Hippocrate 10

B-1200 Brussels

Belgium

Fax: +33-3-83153633

pdeprez@uclouvain.be 